\title{
DIOPHANTINE APPROXIMATION ON HECKE GROUPS
}

\author{
by J. LEHNER
}

\section{Dedicated to Robert Rankin in admiration and respect}

1. Introduction. If $\alpha$ is a real irrational number, there exist infinitely many reduced rational fractions $p / q$ for which

$$
\left|\alpha-\frac{p}{q}\right|<\frac{1}{\sqrt{5} q^{2}},
$$

and $\sqrt{5}$ is the best constant possible. This result is due to A. Hurwitz. The following generalization was proposed in $[2]$. Let $\Gamma$ be a finitely generated fuchsian group acting on $\mathrm{H}^{+}$, the upper half of the complex plane. Let $\mathscr{L}$ be the limit set of $\Gamma$ and $\mathscr{P}$ the set of cusps (parabolic vertices). Assume $\infty \in \mathscr{P}$. Then if $\alpha \in \mathscr{L}-\mathscr{P}$, we have

$$
\left|\alpha-\frac{p}{q}\right|<\frac{1}{k q^{2}},
$$

for infinitely many $p / q \in \Gamma(\infty)$, where $k$ depends only on $\Gamma$. Attention centers on

$$
h=h(\Gamma)=\sup k \text {, }
$$

$k$ running over the set for which (1.2) holds. We call $h$ the Hurwitz constant for $\Gamma$. When $\Gamma=\Gamma(1)$, the modular group, (1.2) reduces to (1.1) and $h(\Gamma(1))=\sqrt{5}$. A proof of $(1.2)$ when $\Gamma$ is horocyclic (i.e., $\mathscr{L}=\mathbb{R}$, the real axis) was furnished by Rankin [4]; he also found upper and lower bounds for $h$. See also [3, pp. 334-5], where the theorem is proved for arbitrary finitely generated $\Gamma$.

In the present paper we consider the Hecke groups

$$
G_{q}=\left\langle\left(\begin{array}{cc}
1 & \lambda_{q} \\
0 & 1
\end{array}\right),\left(\begin{array}{rr}
0 & -1 \\
1 & 0
\end{array}\right)\right\rangle
$$

where

$$
\lambda_{q}=2 \cos \frac{\pi}{q}, \quad q=4,5, \ldots
$$

These are horocyclic groups. It is our object to evaluate, or at least estimate

$$
h\left(G_{q}\right) \equiv h_{q}
$$

The Hecke groups include $G_{3}=\Gamma(1)$, for which $h_{3}=\sqrt{5}$ as stated above, and

$$
G_{\infty}=\left\langle\left(\begin{array}{ll}
1 & 2 \\
0 & 1
\end{array}\right),\left(\begin{array}{rr}
0 & -1 \\
1 & 0
\end{array}\right)\right\rangle,
$$

a subgroup of $\Gamma(1)$, for which $h_{\infty}=2$, as proved by W. T. Scott [6]-see also [4, p. 289]. We shall therefore confine our attention to $q \geq 4$.

Glasgow Math. J. 27 (1985) 117-127. 
Our results are contained in the following theorems.

THEOREM $1 . h_{2 l}=2, l=2,3, \ldots$.

THEOREM 2. $2 \leq h_{2 l-1} \leq\left[\left(\frac{2}{\lambda-1}-\lambda\right)^{2}+4\right]^{1 / 2}$,

$$
\begin{gathered}
\lambda=\lambda_{2 l-1}, \quad l=4,5, \ldots, \\
2 \leq h_{5} \leq 2.036 .
\end{gathered}
$$

2. The lower bound. We wish to show first that

$$
h_{\mathrm{q}} \geq 2, \quad q \geq 4
$$

as proved in [2]. Here we sketch the argument.

From now on we write

$$
\lambda=\lambda_{q}, \quad G=G_{q} .
$$

A fundamental region for $G_{q}$ is the triangle

$$
R_{0}=\left\{z=x+i y: y>0,|x| \leq \lambda / 2, x^{2}+y^{2} \geq 1\right\} .
$$

This may be regarded as a quadrilateral with $i$ as one vertex, the sides being labelled $1,2,2^{\prime}, 1^{\prime}$, starting with the left-hand vertical and proceeding counterclockwise. The sides $1,1^{\prime}$ are conjugate under $G$, and likewise $2,2^{\prime}$. The set of cusps $\mathscr{P}$ is $G(\infty)$, as $\infty$ is the only cusp in $R_{0}$. The elliptic vertices are $i, \rho=\exp \pi i / q$, and $-\bar{\rho}$.

Let $\alpha \in \mathbb{R}-G(\infty)$. The vertical line $L_{\alpha}=L:=\{\alpha+i y, y>0\}$ crosses infinitely many fundamental regions, say $R_{1}, R_{2}, \ldots$ in order. Let $\mu_{i}=L \cap R_{i}$ be the directed segment of $L$ lying in $R_{i}$. The transformation $V_{i}^{-1} \in G$ maps $R_{i}$ on $R_{0}$ and $\mu_{i}$ on the directed arc $\mu_{i}^{\prime}=V_{i}^{-1} \mu_{i}$ lying in $R_{0}$.

LEMMA 1. For each $\alpha \in \mathbb{R}-G(\infty)$ there exist infinitely many $j$, and corresponding points $t_{j}^{\prime}$ on $\mu_{j}^{\prime}$, such that

$$
\operatorname{Im} t_{j}^{\prime}>1 \text {. }
$$

Proof. Consider the sequence of $\mu_{i}^{\prime}$. Each $\mu_{i}^{\prime}$ cuts two sides of $R_{0}$, say $k_{i}, k_{i+1}$ in that order. Denote the conjugate of $k$ by $k^{\prime}$ and set $\left(k^{\prime}\right)^{\prime}=k$. Then $\mu_{i+1}^{\prime}$ cuts $k_{i+1}^{\prime}, k_{i+2}$. Suppose $k_{i}, k_{i+1}$ are not consecutive sides of $R_{0}$; since $\mu_{i}^{\prime}$ (when extended) is orthogonal to $\mathbb{R}$, this means $\left(k_{i}, k_{i+1}\right)=\left(1,2^{\prime}\right),\left(2,1^{\prime}\right),\left(1,1^{\prime}\right)$ or their reverses. Then it is clear there is a $t_{i}^{\prime}$ on $\mu_{i}^{\prime}$ satisfying (2.3). Hence if the lemma is untrue we must assume the sequence of $k$ 's is eventually $k, l ; l^{\prime}, k^{\prime} ; k, l ; \ldots$, where $(k, l)=(1,2),\left(2^{\prime}, 1^{\prime}\right)$, or their reverses. In the first case, for example, this would mean that infinitely many sides of fundamental regions issue from the elliptic vertex $-\bar{\rho}$. This is impossible.

Having proved the lemma, we now write $t_{j}=V_{j} t_{i}^{\prime} \in L \quad(j=1,2, \ldots)$. Thus $t_{j}=$ $\alpha+i y_{j}, j>0$. Let $V_{j}^{-1}=\left(q_{j}^{\prime},-p_{j}^{\prime}: q_{j},-p_{j}\right)$. Then

$$
\operatorname{Im} t_{j}^{\prime}=\frac{y_{i}}{\left(q_{j} \alpha-p_{j}\right)^{2}+q_{i}^{2} y_{j}^{2}}>1 \text {. }
$$


Since the arithmetic mean is not less than the geometric mean, we get

$$
y_{j}>\left(q_{j} \alpha-p_{j}\right)^{2}+q_{j}^{2} y_{j}^{2} \geq 2\left|q_{j} \alpha-p_{j}\right| q_{j} y_{j} \text {. }
$$

Hence

$$
\left|\alpha-\frac{p_{j}}{q_{j}}\right|<\frac{1}{2 q_{j}^{2}} \quad(j=1,2, \ldots),
$$

which implies (2.1).

3. Approximation by $\lambda$-fractions. One of the standard ways of proving Hurwitz's theorem (1.1) is to use regular continued fractions. One first shows that if (1.1) is satisfied by any rational number $P / Q$ in lowest terms, then $P / Q$ must be a convergent in the expansion of $\alpha$ as a regular continued fraction. The problem is thus reduced to studying the approximation of $\alpha$ by its convergents.

We follow a similar route. For a given $\lambda=\lambda_{q}, q \geq 4$, a $\lambda$-fraction ( $\lambda \mathrm{CF}$ ) has been developed by D. Rosen [5]. A $\lambda$ CF is a finite or infinite continued fraction

$$
\left[r_{0} \lambda, \varepsilon_{1} / r_{1} \lambda, \ldots\right]=r_{0} \lambda+\frac{\varepsilon_{1}}{r_{1} \lambda+\ldots},
$$

where the integers $r_{i}, i \geq 1$, are positive and $\varepsilon= \pm 1$. If the fraction is finite, we can write

$$
\left[r_{0} \lambda, \varepsilon_{1} / r_{1} \lambda, \ldots, \varepsilon_{n} / r_{n} \lambda\right]=S^{r_{\circ}} T S^{-\varepsilon_{1} r_{1}} \ldots S^{-\varepsilon_{n} r_{n}} T(\infty),
$$

where $S=(1, \lambda: 0,1), T=(0,-1: 1,0)$ are the generators of $G$. It follows that every finite $\lambda \mathrm{CF}$ has a value that is a cusp of $G$ (belongs to $G(\infty)$ ) and conversely. An infinite convergent $\lambda \mathrm{CF}$, then, converges to a point in $\mathbb{R}-G(\infty)$.

In the $\lambda \mathrm{CF}$ (3.1) define

$$
\begin{gathered}
P_{-1}=1, \quad P_{0}=r_{0} \lambda, \quad Q_{-1}=0, \quad Q_{0}=1, \\
P_{n}=r_{n} \lambda P_{n-1}+\varepsilon_{n} P_{n-2}, \quad Q_{n}=r_{n} \lambda Q_{n-1}+\varepsilon_{n} Q_{n-2}, \quad n \geq 1 .
\end{gathered}
$$

Then we derive the following equations.

$$
\begin{gathered}
{\left[r_{0} \lambda, \varepsilon_{1} / r_{1} \lambda, \ldots, \varepsilon_{n} / r_{n} \lambda\right]=P_{n} / Q_{n}} \\
P_{n} Q_{n-1}-Q_{n} P_{n-1}=(-1)^{n-1} \varepsilon_{1} \varepsilon_{2} \ldots \varepsilon_{n} .
\end{gathered}
$$

If $\alpha$ is represented by the convergent $\lambda$ CF (3.1), we have

$$
\alpha=\lim _{n \rightarrow \infty} P_{n} / Q_{n}
$$

Defining the "tail" of (3.1) by

$$
\alpha_{n}=\left[r_{n} \lambda, \varepsilon_{n+1} / r_{n+1} \lambda, \ldots\right] \quad(n \geq 0),
$$

we get

$$
\alpha=\left[r_{0} \lambda, \ldots, \frac{\varepsilon_{n-1}}{r_{n-1} \lambda}, \frac{\varepsilon_{n}}{\alpha_{n}}\right]
$$


Now this is a finite $\lambda$ CF whose value is $P_{n} / Q_{n}$. Using (3.2) we obtain

$$
\begin{gathered}
\alpha=\frac{\alpha_{n} P_{n-1}+\varepsilon_{n} P_{n-2}}{\alpha_{n} Q_{n-1}+\varepsilon_{n} Q_{n-2}} \quad(n \geq 1) \\
\alpha-\frac{P_{n-1}}{Q_{n-1}}=\frac{(-1)^{n-1} \varepsilon_{1} \varepsilon_{2} \ldots \varepsilon_{n}}{m_{n-1} Q_{n-1}^{2}}
\end{gathered}
$$

with

$$
\begin{aligned}
m_{n-1}(\alpha) & \equiv m_{n-1}=\alpha_{n}+\varepsilon_{n} Q_{n-2} / Q_{n-1} \\
& =\left[r_{n} \lambda, \frac{\varepsilon_{n+1}}{r_{n+1} \lambda}, \ldots\right]+\varepsilon_{n} /\left[r_{n-1} \lambda, \frac{\varepsilon_{n-1}}{r_{n-2} \lambda}, \ldots, \frac{\varepsilon_{2}}{r_{1} \lambda}\right] .
\end{aligned}
$$

Rosen calls (3.1) reduced if certain conditions on $r_{i}$ and $\varepsilon_{i}$ are fulfilled; these will be given later. $\mathrm{He}$ establishes the following properties of reduced $\lambda \mathrm{CF}$.

(3.8) An infinite reduced $\lambda C F$ converges.

(3.9) Every real number $\alpha$ can be expanded uniquely by the "nearest integer algorithm" in a reduced $\lambda C F$. If the fraction is infinite, it converges to $\alpha$.

(3.10) In a reduced $\lambda C F$ we have $Q_{n} \geq 1, Q_{n}$ is non-decreasing, and $Q_{n} \rightarrow \infty$ as $n \rightarrow \infty$.

We denote the expansion of $\alpha$ in a reduced $\lambda \mathrm{CF}$ by $\lambda \mathrm{CF} \alpha$.

REMARK. The $\lambda \mathrm{CF}$ is a type of semi-regular continued fraction, but as the above shows, it possesses properties beyond those of the general semi-regular continued fraction.

We are now ready to prove the following result.

TheOREM 3. For $P / Q \in G(\infty)$ and $\alpha \in \mathbb{R}-G(\infty)$ let

$$
|\alpha-P / Q|<1 / 2 Q^{2} \text {. }
$$

Then $P / Q$ is a convergent of the reduced $\lambda C F \alpha$.

Proof. Write

$$
\alpha-\frac{P}{Q}=\frac{\varepsilon \theta}{Q^{2}}, \quad 0<\theta<\frac{1}{2}, \quad \varepsilon= \pm 1 .
$$

Expand $P / Q$ in a reduced $\lambda \mathrm{CF}$ :

$$
P / Q=\left[r_{0} \lambda, \varepsilon_{1} / r_{i} \lambda, \ldots, \varepsilon_{n-1} / r_{n-1} \lambda\right]
$$

the fraction is finite because $P / Q \in G(\infty)$. Call the convergents $P_{i} / Q_{i}$, so that

$$
\frac{P}{Q}=\frac{P_{n-1}}{Q_{n-1}} \text {. }
$$


Next define $\omega$ by

$$
\alpha=\frac{P_{n-1} \omega+\varepsilon_{n} P_{n-2}}{Q_{n-1} \omega+\varepsilon_{n} Q_{n-2}},
$$

where we have introduced $\varepsilon_{n}$ by

$$
\varepsilon=\varepsilon_{1} \varepsilon_{2} \ldots \varepsilon_{n}(-1)^{n-1}
$$

Then using (3.3) we get

and so

$$
\begin{aligned}
\frac{\varepsilon \theta}{Q_{n-1}^{2}}=\alpha-\frac{P}{Q} & =\frac{P_{n-1} \omega+\varepsilon_{n} P_{n-2}}{Q_{n-1} \omega+\varepsilon_{n} Q_{n-2}}-\frac{P_{n-1}}{Q_{n-1}} \\
& =\frac{\varepsilon_{n} \varepsilon_{1} \varepsilon_{2} \ldots \varepsilon_{n-1}(-1)^{n-1}}{Q_{n-1}\left(Q_{n-1} \omega+\varepsilon_{n} Q_{n-2}\right)},
\end{aligned}
$$

$$
\theta=\frac{Q_{n-1}}{Q_{n-1} \omega+\varepsilon_{n} Q_{n-2}}
$$

Hence

$$
\omega=\frac{Q_{n-1}-\theta \varepsilon_{n} Q_{n-2}}{\theta Q_{n-1}}>0,
$$

because $Q_{n-1} \geq Q_{n-2}$ by $(3.10)-P_{n-1} / Q_{n-1}$ is a reduced $\lambda \mathrm{CF}$-and $0<\theta<1 / 2, \varepsilon_{n}= \pm 1$.

We expand $\omega$ in a reduced $\lambda \mathrm{CF}$

$$
\omega=\left[r_{n} \lambda, \varepsilon_{n+1} / r_{n+1} \lambda, \ldots\right] .
$$

Here $r_{n}=\{\omega / \lambda\}=$ nearest integer to $\omega / \lambda$. Since

$$
\omega=\frac{1}{\theta}-\frac{\varepsilon_{n} Q_{n-2}}{Q_{n-1}}>2-1=1>\frac{\lambda}{2},
$$

it follows that $r_{n} \geq 1$.

We have two expressions for $\alpha$ :

$$
\alpha=\frac{P_{n-1} \omega+\varepsilon_{n} P_{n-2}}{Q_{n-1} \omega+\varepsilon_{n} Q_{n-2}}=\frac{P_{n-1} \alpha_{n}+\varepsilon_{n} P_{n-2}}{Q_{n-1} \alpha_{n}+\varepsilon_{n} Q_{n-2}}
$$

see (3.13), (3.5). Hence $\omega=\alpha_{n}$ and by (3.4), (3.14),

$$
\alpha=\left[r_{0} \lambda, \frac{\varepsilon_{1}}{r_{1} \lambda}, \ldots, \frac{\varepsilon_{n-1}}{r_{n-1} \lambda}, \frac{\varepsilon_{n}}{\alpha_{n}}\right]=\left[r_{0} \lambda, \ldots, \frac{\varepsilon_{n-1}}{r_{n-1} \lambda}, \frac{\varepsilon_{n}}{r_{n} \lambda}, \frac{\varepsilon_{n+1}}{r_{n+1} \lambda}, \ldots\right] .
$$

It follows from (3.12) that

$$
\frac{P}{Q}=\frac{P_{n-1}}{Q_{n-1}}=\left[r_{0} \lambda, \frac{\varepsilon_{1}}{r_{1} \lambda}, \ldots, \frac{\varepsilon_{n-1}}{r_{n-1} \lambda}\right],
$$

and so $P / Q$ is a convergent of $\alpha$. 
4. Theorem 3 permits us to confine our considerations to approximations of $\alpha$ by the convergents of its reduced $\lambda \mathrm{CF}$. By (3.6) we have

$$
\left|\alpha-\frac{P_{n-1}}{Q_{n-1}}\right|=\frac{1}{m_{n-1} Q_{n-1}^{2}},
$$

$m_{n-1} \equiv m_{n-1}(\alpha)$ being given by (3.7). Let

$$
M(\alpha)=\varlimsup_{n \rightarrow \infty} m_{n-1}(\alpha)
$$

then we have

$$
h_{\mathrm{q}}=\inf _{\alpha} M(\alpha), \quad \alpha \in \mathbb{R}-G_{\mathrm{q}}(\infty) \text {. }
$$

In this section we treat the case $q=2 l$, where $l \geq 2$. To prove Theorem 1 , it is obviously sufficient to exhibit one $\alpha$, say $\alpha_{0}$, for which

$$
M\left(\alpha_{0}\right) \leq 2 \text {, }
$$

for this would imply $h_{q} \leq 2$ and we already know $h_{q} \geq 2$ from (2.1).

At this point we introduce the definition of a reduced $\lambda \mathrm{CF}$ when $q=2 l$. Let

$$
s=[(q-3) / 2]=l-2 \geq 0 .
$$

(4.3) The fraction $\left[r_{0} \lambda, \varepsilon_{1} / r_{1} \lambda, \ldots\right]$ is said to be reduced $([5, \mathrm{p} .555])$ if the inequality $r_{i} \lambda+\varepsilon_{i+1}<1$ is satisfied for no more than $s$ consecutive values of $i$, say $i=j, j+1, \ldots, j+s-1 ; j \geq 1$.

This inequality is equivalent to $r_{i}=1, \varepsilon_{i+1}=-1$.

We shall define $\alpha_{0}$ as a (pure) periodic $\lambda$ CF. The infinite $\lambda$ CF $\left[r_{0} \lambda, \varepsilon_{1} / r_{1} \lambda, \ldots\right]$ is periodic if for some integer $p \geq 1$ (the period) and $\nu \geq 0$ we have

$$
r_{\nu+p}=r_{\nu}, \quad \varepsilon_{\nu+p+1}=\varepsilon_{\nu+1} .
$$

We shall write the periodic fraction as

$$
\left[r_{0} \lambda, \frac{\varepsilon_{1}}{r_{1} \lambda}, \ldots, \frac{\varepsilon_{p-1}}{r_{p-1} \lambda}, \frac{\varepsilon_{p}}{r_{0} \lambda}, \frac{\varepsilon_{1}}{r_{1} \lambda}, \ldots\right]=\left[\overline{r_{0} \lambda, \ldots, \frac{\varepsilon_{p-1}}{r_{p-1} \lambda}} ; \frac{\varepsilon_{p}}{r_{0} \lambda}\right] .
$$

Note that it is necessary to exhibit the term $\varepsilon_{\mathrm{p}} / r_{0} \lambda$, since (4.4) does not distinguish between $\varepsilon_{p}=1$ and $\varepsilon_{p}=-1$. The tails of a periodic $\lambda$ CF are also periodic:

$$
\alpha_{0}=\alpha_{p}=\alpha_{2 p}=\ldots
$$

Now consider the periodic $\lambda \mathrm{CF}$

$$
\alpha_{0}=\left[\overline{2 \lambda,-\frac{1}{\lambda}, \ldots,-\frac{1}{\lambda}} ; \frac{-1}{2 \lambda}\right]=\alpha_{s+1}=\ldots
$$

of period $s+1$. This fraction satisfies (4.3) and so is reduced. By (3.8) it converges. Along 
with $\alpha_{0}$ we introduce the reduced $\lambda \mathrm{CF}$

$$
\begin{aligned}
& \alpha_{1} \equiv \beta_{0}=\left[\overline{\lambda,-\frac{1}{\lambda}, \ldots,-\frac{1}{2 \lambda}} ;-\frac{1}{\lambda}\right]=\beta_{s+1}=\ldots, \\
& \alpha_{0}=2 \lambda-1 / \beta_{0} .
\end{aligned}
$$

Let

$$
\alpha_{n}^{\prime}=\left[r_{n} \lambda, \frac{\varepsilon_{n}}{r_{n-1} \lambda}, \ldots, \frac{\varepsilon_{2}}{r_{1} \lambda}\right]
$$

so that by (3.7)

$$
m_{n-1}=\alpha_{n}+\varepsilon_{n} / \alpha_{n-1}^{\prime} .
$$

In the present case, with $p=s+1$, we can write

$$
\begin{gathered}
m_{n p-1}=\alpha_{n p}+\varepsilon_{n p} / \alpha_{n p-1}^{\prime}=\alpha_{0}-1 / \alpha_{n p-1}^{\prime} \\
\lim _{n \rightarrow \infty} m_{n p-1}=\alpha_{0}-1 / \beta_{0}=\alpha_{0}+\alpha_{0}-2 \lambda=2\left(\alpha_{0}-\lambda\right)
\end{gathered}
$$

using (4.9).

We shall prove that

$$
\alpha_{0}=\lambda+1
$$

so that

$$
\lim _{n \rightarrow \infty} m_{n p-1}=2 \text {. }
$$

On the other hand, if $j \neq 0(\bmod p)$,

$$
m_{j-1}=\alpha_{j}-1 / \alpha_{n-1}^{\prime}<\alpha_{j}<\lambda<2=\lim _{n \rightarrow \infty} m_{n p-1} .
$$

Hence (4.2) follows, and with it, Theorem 1.

It remains to prove (4.12). From (3.5) and (4.8) we get

$$
\begin{gathered}
\beta_{0}=\frac{P_{s} \beta_{0}-P_{s-1}}{Q_{s} \beta_{0}-Q_{s-1}}, \\
Q_{s} \beta_{0}^{2}-\left(Q_{s-1}+P_{s}\right) \beta_{0}+P_{s-1}=0,
\end{gathered}
$$

$P_{i} / Q_{i}$ being the convergents of $\beta_{0}$. The value of $Q_{i}$ can be found in [1, p. 7] or is easily checked by induction:

$$
Q_{i}=\frac{\sin (i+1) \pi / q}{\sin \pi / q} \quad(i \leq s-1)
$$


so $(s=l-2)$

$$
\begin{aligned}
& Q_{s-1}=\frac{\sin ((l-2) \pi / 2 l}{\sin \pi / 2 l}=\frac{\cos \pi / l}{\sin \pi / 2 l} \quad(l \geq 2), \\
& Q_{s-2}=\frac{\sin ((l-3) \pi / 2 l}{\sin \pi / 2 l}=\frac{\cos 3 \pi / 2 l}{\sin \pi / 2 l}
\end{aligned}
$$

By induction from (3.2),

$$
P_{i}=Q_{i+1} \quad(i \leq s-2)
$$

Hence

$$
\begin{aligned}
P_{s-1} & =\lambda P_{s-2}-P_{s-3}=\lambda Q_{s-1}-Q_{s-2}=Q_{s}-\lambda Q_{s-1}, \\
P_{s} & =2 \lambda P_{s-1}-P_{s-2}=2 \lambda\left(Q_{s}-\lambda Q_{s-1}\right)-Q_{s-1} \\
& =2 \lambda Q_{s}-\left(2 \lambda^{2}+1\right) Q_{s-1}, \\
Q_{s-1}+P_{s} & =2 \lambda\left(Q_{s}-\lambda Q_{s-1}\right) .
\end{aligned}
$$

We shall shortly see that $Q_{s} \neq 0$. Substituting these values in (4.3) and dividing by $Q_{s}$, we get

$$
\beta_{0}^{2}-2 \lambda\left(1-\lambda \frac{Q_{s-1}}{Q_{s}}\right) \beta_{0}+1-\lambda \frac{Q_{s-1}}{Q_{s}}=0 .
$$

Now with $\zeta=\exp (\pi i / 2 l), \lambda=\zeta+\zeta^{-1}$,

$$
\begin{aligned}
Q_{s}=2 \lambda Q_{s-1}-Q_{s-2} & =\frac{2\left(\zeta+\zeta^{-1}\right)\left(\zeta^{2}+\zeta^{-2}\right)-\left(\zeta^{3}+\zeta^{-3}\right)}{2 \sin \pi / 2 l} \\
& =\frac{\lambda\left(\lambda^{2}-1\right)}{2 \sin \pi / 2 l} \neq 0 \\
\frac{Q_{s-1}}{Q_{s}} & =\frac{\lambda^{2}-2}{\lambda\left(\lambda^{2}-1\right)}
\end{aligned}
$$

Thus (4.14) becomes

$$
\beta_{0}^{2}-\frac{2 \lambda}{\lambda^{2}-1} \beta_{0}+\frac{1}{\lambda^{2}-1}=0
$$

the roots of which are $\beta_{0}=1 /(\lambda-1), 1 /(\lambda+1)$. This gives $\alpha_{0}=\lambda+1, \lambda-1$. However, from the definition (4.7), $\alpha_{0}>2 \lambda-1>\lambda-1$, and so we have proved (4.12). Theorem 1 is now established.

5. We turn now to the case $q$ odd, $q>5$ :

$$
q=2 l-1, \quad l \geq 4 ; \quad s=l-2 .
$$

There are additional conditions besides (4.3) in the definition of an infinite reduced $\lambda \mathrm{CF}$ when $q$ is odd. 
(5.1) If $r_{i} \lambda+\varepsilon_{i+1}<1$ for $i=j, j+1, \ldots, j+s-1$, then $r_{j+s} \geq 2$.

(5.2) If $(B(s),-1 / 2 \lambda,-1 / B(s))$ occurs in the expansion, the succeeding sign is plus. Here

$$
B(n)=\left(\lambda,-\frac{1}{\lambda}, \ldots,-\frac{1}{\lambda}\right)
$$

with $n$ partial quotients.

One way to avoid these rather complicated conditions is to use fractions with less than the maximum allowable number of consecutive sequences $r_{i} \lambda+\varepsilon_{i+1}<1$. For example, let

$$
\alpha_{0}=\left[\overline{\lambda,-\frac{1}{\lambda}, \ldots,-\frac{1}{\lambda}} ; \frac{1}{\lambda}\right]=\lim _{n \rightarrow \infty} \frac{P_{n}}{Q_{n}}
$$

of period $s$, in which there are $s-1$ consecutive sequences. Then

$$
\alpha_{0}=\alpha_{s}=\frac{P_{s-1} \alpha_{0}+P_{s-2}}{Q_{s-1} \alpha_{0}+Q_{s-2}} .
$$

A calculation quite similar to that of Section 4 yields

$$
\alpha_{0}^{2}+\left(\lambda-\frac{2}{\lambda-1}\right) \alpha_{0}-1=0
$$

where we have used

$$
Q_{s-1}=\frac{\sin (s \pi /(2 l-1))}{\sin (\pi /(2 l-1))}=\frac{\cos (3 \pi / 2(2 l-1))}{\sin (\pi /(2 l-1))} \neq 0 .
$$

Continuing,

$$
\begin{aligned}
\alpha_{n s-1}^{\prime} & =\left[\lambda,-\frac{1}{\lambda}, \ldots,-\frac{1}{\lambda}, \frac{1}{\lambda},-\frac{1}{\lambda}, \ldots, \frac{1}{\lambda}\right] \\
& \rightarrow\left[\lambda,-\frac{1}{\lambda}, \ldots,-\frac{1}{\lambda} ; \frac{1}{\lambda}\right]=\alpha_{0} .
\end{aligned}
$$

Hence

$$
m_{n s-1} \rightarrow \alpha_{0}+\frac{1}{\alpha_{0}}, \quad \text { as } \quad n \rightarrow \infty
$$

Now

$$
\left(\alpha_{0}+1 / \alpha_{0}\right)^{2}=\left(\alpha_{0}-\bar{\alpha}_{0}\right)^{2}=\left(\alpha_{0}+\bar{\alpha}_{0}\right)^{2}-4 \alpha_{0} \bar{\alpha}_{0}=\left(\frac{2}{\lambda-1}-\lambda\right)^{2}+4,
$$

$\bar{\alpha}_{0}$ being the other root of (5.4). So we get

$$
\lim _{n \rightarrow \infty} m_{n s-1}=M_{q}
$$


where

$$
M_{\mathrm{q}}=\alpha_{0}+\frac{1}{\alpha_{0}}=\left[\left(\frac{2}{\lambda-1}-\lambda\right)^{2}+4\right]^{1 / 2} .
$$

Again, for $j \neq 0,1(\bmod s), m_{j-1}=\alpha_{j}-1 / \alpha_{j-1}^{\prime}<\alpha_{j}<2<M_{\mathrm{q}}$. For $j=n s-1$,

$$
\begin{aligned}
m_{j-1}=m_{n s-2} & =\alpha_{n s-1}-1 / \alpha_{n s-2}^{\prime} \\
& =\lambda+\frac{1}{\alpha_{s}}+\alpha_{n s-1}^{\prime}-\lambda \rightarrow \frac{1}{\alpha_{0}}+\alpha_{0}=M_{\mathrm{q}} .
\end{aligned}
$$

It follows that

$$
h_{21-1} \leq M\left(\alpha_{0}\right) \leq M_{q}
$$

as asserted in Theorem 2. For example, $M_{7} \approx 2 \cdot 12$. We observe that $M_{q} \rightarrow 2$ as $q \rightarrow \infty$.

The final case, $q=5(l=3)$, can be treated in the same way, the result being $M_{5} \approx 2 \cdot 57$. We can get a better result, however, by taking

$$
\alpha_{0}=\left[\overline{\lambda,-\frac{1}{2 \lambda},-\frac{1}{2 \lambda}} ;-\frac{1}{\lambda}\right]
$$

of period 3, which is reduced. The equation for $\alpha_{0}$ is

$$
\alpha_{0}^{2}-\lambda \alpha_{0}+\frac{2 \lambda-1}{5}=0
$$

where we have replaced the rational functions of $\lambda$ that occur by polynomials, using $\lambda^{2}-\lambda-1=0, \lambda=\lambda_{5}=(1+\sqrt{5}) / 2$. The roots of $(5.7)$ are $(1 \cdot 264,0 \cdot 354), \alpha_{0}$ being equal to the larger one. Now

$$
\begin{gathered}
m_{3 n}=\alpha_{3 n+1}-\frac{1}{\alpha_{3 n}^{\prime}}=\alpha_{1}-\frac{1}{\alpha_{0}}=\frac{1}{\lambda-\alpha_{0}}-\frac{1}{\alpha_{0}}, \\
m_{3 n+1}=\alpha_{3 n+2}-\frac{1}{\alpha_{3 n+1}^{\prime}} \rightarrow \alpha_{2}-\frac{1}{\alpha_{2}}, \alpha_{2}=2 \lambda-\frac{1}{\alpha_{0}}, \\
m_{3 n+2}=\alpha_{3 n+3}-\frac{1}{\alpha_{3 n+2}^{\prime}} \rightarrow \alpha_{0}-\frac{1}{\alpha_{1}}=2 \alpha_{0}-\lambda .
\end{gathered}
$$

Calculation shows that the largest of the right members is

$$
\lim m_{3 n+1} \approx 2 \cdot 036 \text {, }
$$

which gives the upper bound for $h_{5}$ in Theorem 2 .

\section{REFERENCES}

1. A. Guillet et M. Aubert, Propriétés des Polynomes Electrosphériques, Memorial des Sciences Mathematiques, Fasc. 107. 
2. J. Lehner. A diophantine property of Fuchsian groups, Pacific J. Math. 2 (1952), 327-333.

3. J. Lehner. Discontinuous groups and automorphic functions, Surveys No. 8 (Amer. Math. Soc., Providence, 1964).

4. R. A. Rankin. Diophantine approximation and horocyclic groups, Canad. J. Math. 9 (1957), 277-290.

5. D. Rosen. A class of continued fractions associated with certain properly discontinuous groups, Duke Math. J. 21 (1954), 549-562.

6. W. J. Scott. Approximation to real irrationals by certain classes of rational fractions, Bull. Amer. Math. Soc. 46 (1940), 124-129.

INSTTTUTE FOR ADVANCED STUDY

Princeton, NeW Jersey 08540

U.S.A. 